\title{
Identification of three new mutations of the HNF-1 $\alpha$ gene in Japanese MODY families
}

\author{
T. Ikema, Y. Shimajiri, I. Komiya, M. Tawata, S. Sunakawa, H. Yogi, M. Shimabukuro, N. Takasu \\ Second Department of Internal Medicine, University of the Ryukyus School of Medicine, Nishihara, Okinawa, Japan
}

\begin{abstract}
Aim/hypothesis. We analysed Japanese MODY patients for mutations in the $H N F-1 \alpha$ gene.

Methods. Fifty unrelated Japanese patients with earlyonset diabetes (diagnosed at 25 years of age or younger) or with a strong family history of diabetes were screened for mutations in the $H N F-1 \alpha$ gene. Functional studies of the mutant HNF-1 $\alpha$ were carried out. Results. We identified three new mutations in the $H N F-1 \alpha$ gene in the families with a strong family history for diabetes. One mutation (L518P519fsTCC $\rightarrow A$ ) was identified in three unrelated families, while the other two mutations (T521I and V617I) were identified in one family. We also identified the A site of the promoter $(+102 G-t o-C)$, which was reported previously. We examined the functional properties of the mutant HNF-1 $\alpha$. By increasing the amount of L518P519fsTCC $\rightarrow$ A-HNF-1 $\alpha$, increasing inhibition
\end{abstract}

of the transcription of human transthyretin (TTR) was observed (up to $61 \%$ of the control). Increasing amounts of T521I-HNF- $1 \alpha$ or V617I-HNF- $1 \alpha$ mutant proteins increased TTR promoter transcription up to 4.3-fold and 2.4-fold, respectively, whereas both increased transcription up to 12.4-fold of the control.

Conclusion/interpretation. The L518P519fsTCC $\rightarrow A$ was identified for the first time and this mutation might be a common cause of Japanese MODY3 in Okinawa area. In addition, both the T521I and V617I mutations were present in two patients in the same family. Since the prevalence of these mutations is relatively high $(10 \%, 5 / 50)$, the $H N F-1 \alpha$ gene needs to be screened for mutations in patients either with earlyonset diabetes or with a strong family history for diabetes. [Diabetologia (2002) 45:1713-1718]

Keywords MODY, HNF-1 $\alpha$, mutation, Japanese, gain-of-function.
Maturity-onset diabetes of the young is a monogenic form of diabetes mellitus characterised by an autosomal dominant inheritance, early-onset (usually before 25 years of age), and impaired insulin secretion [1].

Received: 8 April 2002 / Revised: 15 July 2002

Published online: 12 November 2002

C) Springer-Verlag 2002

Corresponding author: Dr. I. Komiya, Second Department of Internal Medicine, University of the Ryukyus School of Medicine, 207 Uehara, Nishihara, Okinawa 903-0215, Japan. E-mail: ikom.iya@k3.dion.ne.jp

Abbreviations: TTR, Transthyretin; PKL, L-type pyruvate kinase; WT, wild type; SPIDDM, slowly progressive insulin dependent diabetes mellitus; OHA, oral hypoglycaemic agents; MEN, multiple endocrine neoplasia.
The most commonly-identified cause of MODY in most racial groups is mutations in the $H N F-1 \alpha$ gene (MODY3).

HNF- $1 \alpha$ is a homeodomain-containing transcription factor which is most highly expressed in the liver, kidneys, intestines, and pancreas. It is composed of three functional domains: an N-terminal dimerization domain (amino acids 1 to 32), a DNA binding domain (amino acids 150 to 280) with POU-like and homeodomain motifs, and a C-terminal transactivation domain (amino acids 281 to 631). Although the function of HNF-1 $\alpha$ in the pancreatic beta cell is less clear, it has been reported to have some effects on the in vitro transcription of the rat insulin I gene [2]. It could also regulate the expression of several genes involved in the uptake and metabolism of glucose by the beta cell. 
The glucose transporter 2 (GLUT2) gene has an HNF$1 \alpha$ binding site in its promoter [3]. The expression of the L-type pyruvate kinase (PKL), which is also present in pancreatic islets and insulinoma cells, is regulated in part by HNF-1 $\alpha[4,5,6]$.

We analysed the $H N F-1 \alpha$ gene in 50 Japanese patients with early-onset diabetes or with a strong family history for diabetes. We identified three novel mutations in the $H N F-1 \alpha$ gene and examined the functional properties of the mutant HNF-1 $\alpha$.

\section{Materials and methods}

Subjects and screening of HNF-1 $\alpha$ gene. We studied 50 unrelated Japanese patients with early-onset diabetes (diagnosed at 25 years of age or younger) or with a strong family history of diabetes. We enrolled 100 non-diabetic subjects without a family history for diabetes ( $>60$ years of age). Nondiabetic subjects were confirmed to have fasting plasma glucose concentrations of less than $6.1 \mathrm{mmol} / \mathrm{l}$ and $\mathrm{HbA}_{1 \mathrm{c}}$ of less than $5.8 \%$ [7]. Informed consent was obtained from all subjects. The study was approved by the Ethics Committee of University of the Ryukyus School of Medicine and was carried out in accordance with the Declaration of Helsinki as revised in 2000. The 10 exons, flanking introns, and minimal promoter region were screened for mutations by polymerase chain reaction (PCR) amplification and direct sequencing of the PCR products as described previously [8].

Preparation of DNA constructs and functional study of the mutant HNF-1 $\alpha$ gene. Human HNF-1 $\alpha$ complementary DNA (cDNA) cloned in the pcDNA3.1/HisC expression vector was provided by Dr. K. Yamagata (Osaka University School of Medicine, Osaka, Japan). A $251 \mathrm{bp}$ fragment of the human transthyretin $(T T R)$ gene promoter $(-218$ to +33 relative to the cap site) was subcloned into the SmaI site of pGL3 basic luciferase reporter plasmid (Promega, Madison, Wiss., USA) provided by Dr. K. Yamagata. Mutagenesis was done by using the Quick Change Site-Directed Kit (Stratagene, La Jolla, Calif., USA) with $P f u$ polymerase and the restriction enzyme Dpn I to generate mutant cDNAs.
Transactivation activity. COS-7 cells were maintained in DMEM supplemented with $10 \%$ fetal bovine serum. The reporter plasmid (TTR-pGL3) was cotransfected with wild type (WT)- $H N F$ - $1 \alpha$-pcDNA3.1/HisC, mutant- $H N F-1 \alpha$-pcDNA3.1/ HisC and pcDNA3.1/HisC vector into COS-7 cells $\left(8 \times 10^{4}\right.$ per well in 24-well tissue culture dishes) using Effectene transfection reagent (QIAGEN, Hilden, Germany). The cells were cultured for $48 \mathrm{~h}$, and the transient luciferase activity was measured. The transfection efficiencies were normalised using Renilla luciferase activity cotransfected in each experiment.

Statistical analysis. Results are expressed as the mean \pm SD. The data from the luciferase reporter assays were analysed by Student's $t$ test. A $p$ value less than 0.05 was considered statistically significant.

\section{Results}

Identification of three novel mutations. We identified a novel frameshift mutation that results from the simultaneous insertion of an adenine base and the deletion of a triplet TCC with a net $2 \mathrm{bp}$ deletion (L518P519fsTCC $\rightarrow A$ ), and two novel missense mutations, T521I and V617I. The frameshift mutation was predicted to cause premature termination at codon 548. We also identified a conserved $\mathrm{G}$ to $\mathrm{C}$ replacement in the A site of the promoter (designated as $+102 G$-to- $C$, relative to the transcription start site) which was reported previously [9]. None of these three mutations were found in the 100 control nondiabetic subjects.

Clinical profiles of subjects with mutation. The L518P519fsTCC $\rightarrow A$ mutation was found in three unrelated families in this study. The clinical features of the six subjects with the L518P519fsTCC $\rightarrow$ A mutation are shown in Table 1 .

In family $\mathrm{A}$ the proband is a 20 -year-old man who was diagnosed with insulin-dependent diabetes (IDDM) at 1 year of age and was treated with insulin.

Table 1. Clinical characteristics of Japanese subjects with the L518P519fsTCC $\rightarrow A$ mutation

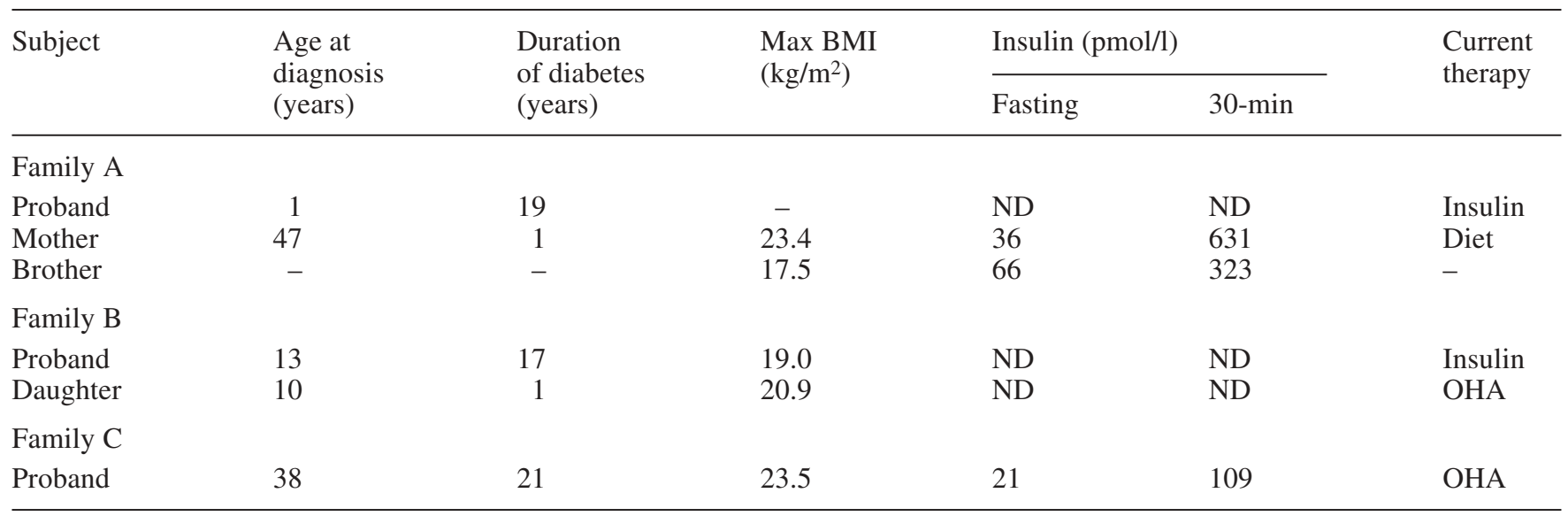

$O H A$ oral hypoglycaemic agent, $N D$ no data 
A

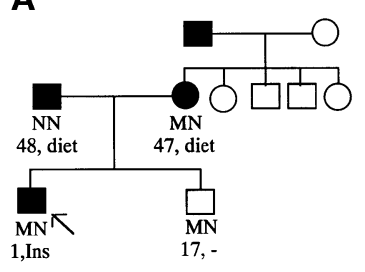

D

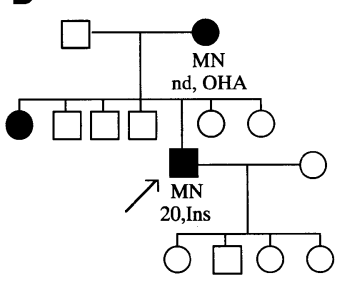

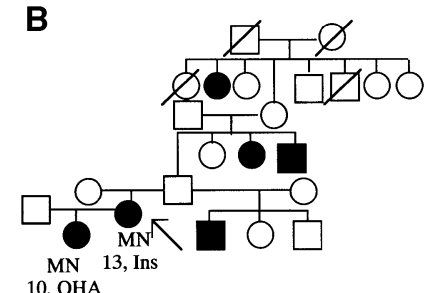

E

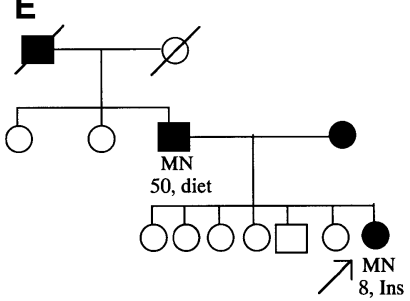

C

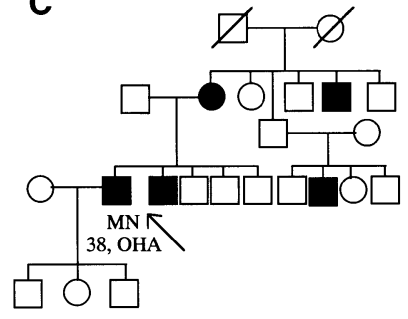

Fig. 1A-E. Pedigree of the family with L518P519fsTCC $\rightarrow A$ $(\mathbf{A}, \mathbf{B}$, and $\mathbf{C})$, both T521I and V617I (D), the A site of the $H N F-1 \alpha$ promoter $+102 G$-to- $C$ (E) mutation in the $H N F-1 \alpha$ gene. Solid symbols: subjects with diabetes, open symbols: non-diabetic subjects. The arrows indicate the probands. The $H N F-1 \alpha$ genotype of each subject tested is indicated below the symbol: $N$, normal; $M$, mutant. The age of diagnosis and treatment (Ins, insulin; OHA, oral hypoglycaemic agent) or age of testing $75 \mathrm{~g}$ OGTT is shown below the genotype information

There were no antibodies to glutamic acid decarboxylase $\left(\mathrm{GAD}_{65}\right)$ in his stored serum. He had proliferative retinopathy and nephropathy and underwent a kidney transplantation at 16 years of age. The L518P519fsTCC $\rightarrow A$ mutation was found in his mother and his younger brother (Fig. 1A). His mother had overt diabetes with postprandial hyperinsulinaemia (fasting IRI, $36 \mathrm{pmol} / \mathrm{l} ; 30 \mathrm{~min}$ IRI, $631 \mathrm{pmol} / \mathrm{l}$ ) as measured during a $75 \mathrm{~g}$ OGTT. His brother had a normal glucose tolerance test with a normal insulin secretory response (fasting IRI, $66 \mathrm{pmol} / \mathrm{l} ; 30 \mathrm{~min}$ IRI, $323 \mathrm{pmol} / \mathrm{l}$ ) at the time of examination (Table 1).

In family B the proband is a 29-year-old woman. She was diagnosed with diabetes at 13 years of age and was treated with an oral hypoglycaemic agent (OHA). Her stored serum was negative for anti$\mathrm{GAD}_{65}$. When she was pregnant at 18 years of age she started insulin therapy. Her BMI was $19.0 \mathrm{~kg} / \mathrm{m}^{2}$ at the onset of diabetes and was therefore initially diagnosed as having a slowly-progressive insulin-dependent diabetes mellitus (SPIDDM) [10]. Her daughter was diagnosed with diabetes mellitus with ketoacidosis at 10 years of age. Her BMI was $20.9 \mathrm{~kg} / \mathrm{m}^{2}$ at the onset of the diabetes. She was treated with OHA at 6 months after diagnosis. The L518P519fsTCC $\rightarrow$ A mutation was also identified in the proband's daughter. Her aunts, uncle and younger brother all had diabetes mellitus but blood samples were not available for the genotyping of the $H N F-1 \alpha$ gene (Fig. 1B).

In family $\mathrm{C}$ the proband, carrying the mutation L518P519fsTCC $\rightarrow$ ' is a 59-year-old man diagnosed as having diabetes at 38 years of age and was treated with diet. His serum was negative for the anti-GAD 65 antibody. OHA treatment was started 16 years after the initial diagnosis. His present $\mathrm{HbA}_{1 \mathrm{c}}$ is $6.0 \%$, about $6.5 \%$ on OHA treatment. His mother, brother, aunt, and cousin had diabetes, but their DNA was not available for the genotyping of the $H N F-1 \alpha$ genes (Fig. 1C).

In the proband, carrying T521I and V617I mutations, is a 58-year-old man. He was diagnosed as having diabetes at 20 years of age and was treated with OHA. His serum was negative for the anti-GAD 65 antibody. Insulin treatment was started 20 years after the initial diagnosis. His mother had diabetes and was found to have the same two missense mutations (Family D, Fig. 1D).

In family $\mathrm{E}$ the proband is a 24 -year-old woman diagnosed with Type I (insulin-dependent) diabetes mellitus at 8 years of age and was treated with insulin. Her serum was positive for the anti-GAD 65 antibody. Her father had overt diabetes and the identical mutation was found. Her mother was also diagnosed as having Type II diabetes and was treated with OHA but her DNA was not available for the genotyping of the $H N F-1 \alpha$ gene. The siblings of the proband were not available for the measurement of plasma glucose, $\mathrm{HbA}_{1 \mathrm{c}}$, or DNA examination (Family E, Fig. 1E).

Functional analysis of the mutant HNF-1 $\alpha$ gene. The transactivation activity of L518P519fsTCC $\rightarrow$ A was $4 \%$ of that of WT in the COS-7 cell (Fig. 2A). Increasing amounts of mutated-HNF-1 $\alpha$ inhibited up to $61 \%$ of the control luciferase activity of a reporter TTR-pGL3 plasmid in a dose-dependant manner (Fig. 3A), suggesting that L518P519fsTCC $\rightarrow$ A functions in a dominant negative manner.

The transactivation activities of T521I, V617I, and both were $47 \%, 63 \%$, and $30 \%$ of that of WT in COS-7 cell, respectively (Fig. 2B). Increasing amounts of T521I-HNF-1 $\alpha$ (Fig. 3B), V617I-HNF-1 $\alpha$ (Fig. 3C), and both (Fig. 3D) mutants, increased up to 4.3-fold, 2.4-fold, and 12.4-fold of the control luciferase activity of a reporter TTR-pGL3 plasmid, respectively. 

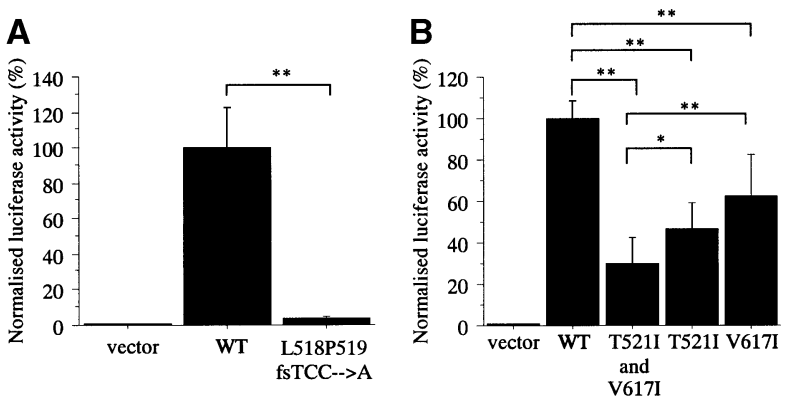

Fig. 2A, B. Transcriptional activity of TTR in COS-7 cell. Seventy-five nanograms of WT, L518P519fsTCC $\rightarrow$ A (A), T521I (B), V617I (B), T521I and V617I (B)-HNF-1 $\alpha$ -pcDNA3.1/HisC DNA and empty vector were transfected with $100 \mathrm{ng}$ of TTR-reporter gene. Data are the means \pm SD of eight independent experiments. $* p<0.05 * * p<0.01$
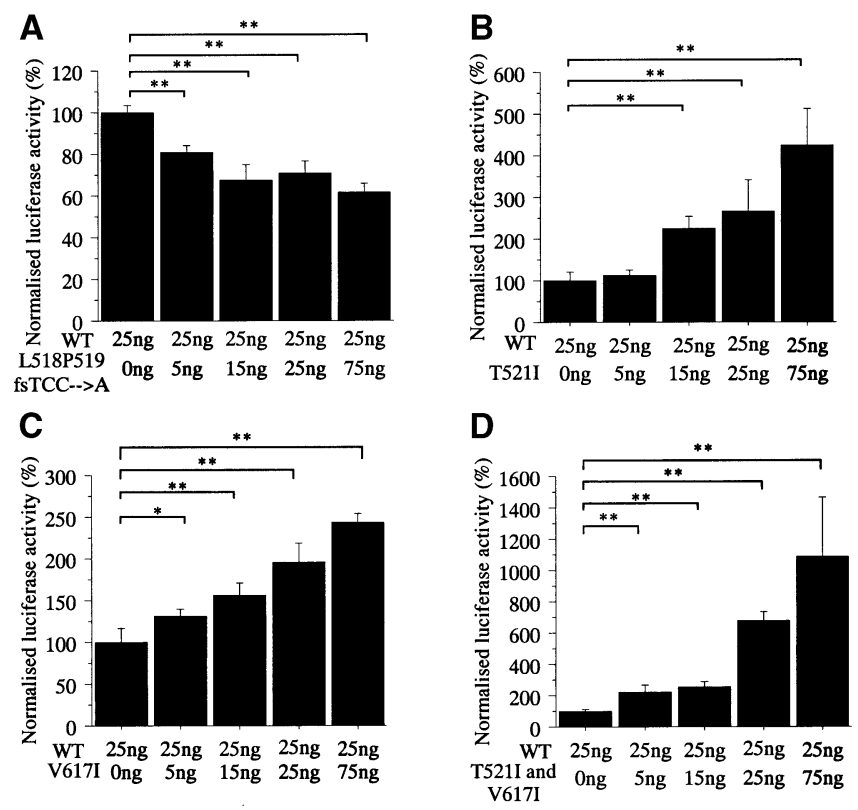

Fig. 3A-D. Transcriptional activity of TTR in COS-7 cell. Twenty-five nanograms of WT-HNF-1 $\alpha$-pcDNA3.1/HisC DNA were transfected alone or with increasing amounts $(5,15,25$, and $75 \mathrm{ng}$ ) of the L518P519fsTCC $\rightarrow$ A (A), T521I (B), V617I (C), T521I and V617I (D)- HNF-1 $\alpha$-pcDNA3.1/HisC DNA. The total amount of DNA added was adjusted to a total of $200 \mathrm{ng}$ using empty pcDNA3.1/HisC DNA. Data are the means $\pm \mathrm{SD}$ of eight independent experiments. ${ }^{*} p<0.05$. $* * p<0.01$

We also investigated functional studies of these mutants in INS1 cells. The results were almost similar with those of COS-7 cells.

\section{Discussion}

In the present study, we identified four different mutations in the $H N F-1 \alpha$ gene in five cases out of 50 Japanese patients with early-onset diabetes or with a strong family history of diabetes. The overall prevalence of the $H N F-1 \alpha$ gene mutation was $10 \%$ in our study in the Okinawa area.
We identified a $+102 G$-to- $C$ mutation, which has been reported previously [9]. This mutation was found in a diabetic patient, who was diagnosed as having diabetes at 8 years of age and was anti-GAD-positive. Patients with anti-beta cell antibodies are characterised by a younger age at diagnosis, a lower body weight, and a higher prevalence of insulin treatment than antibody-negative patients within identical MODY3 families [11]. We also identified a novel L518P519fsTCC $\rightarrow$ A mutation in three unrelated families. The P291fsins $C$ mutation is the most common cause of MODY3 in Caucasian subjects [12, 13, 14]. In 60 MODY probands, eight (13\%) had the $P 291$ sinsC mutation [14]. The L518P519fsTCC $\rightarrow A$ mutation might be a common cause of Japanese MODY3 (6\%) in the Okinawa area. The presence of two mutations in the $H N F-1 \alpha$ gene in one patient has not been reported so far. However, we identified the T521I and V617I-HNF-1 $\alpha$ mutations both present in two related subjects.

Concerning the functional properties of these mutations, the promoter activity of the $+102 G$-to- $C$ construct was increased by $40 \%$ and $80 \%$ compared with that of a WT-promoter construct in HepG2 and MIN6 cell, respectively [9]. The authors discussed that the $+102 G$-to- $C$ mutation possibly leads to increased promoter activity and thus might lead to higher than normal concentrations of HNF- $1 \alpha$ protein and activity [9].

The functional studies indicate that the L518P519fsTCC $\rightarrow$ A mutation has decreased transactivation activity and acts on HNF-1 $\alpha$ in a dominant negative manner. However, functional studies of T521I and V617I mutations under the coexistence of wild type HNF-1 $\alpha$ showed rather unexpected results, suggesting gain-of-function mutants. The R203C mutation in the $H N F-1 \alpha$ gene has been reported [15]. The functional analysis of the R203C mutation showed that the mutant alone increased the transactivation of the GLUT2 promoter, whereas the coexistence of the wild type $H N F-1 \alpha$ and the mutant inhibited transactivation [15]. Therefore we think that it is possible to get these unexpected results by unknown mechanisms.

It has been known that loss-of-function and gainof-function mutations in the same gene have different pathologic effects. For example, gain-of-function ret is caused by gene rearrangements in thyroid papillary carcinomas and by point mutations in multiple endocrine neoplasia (MEN) in type IIA (MEN IIA), in familial medullary thyroid carcinoma, and in MEN IIB [16]. Conversely, Hirschsprung's disease, frequently accompanied with MEN IIB, is associated with lossof-function of ret [16]. Thus, the T521I and V617I mutations could be similar to the $+102 \mathrm{G}$-to-C in the promoter region, in which the mutations are expected to result in an overall increase in HNF- $1 \alpha$ activity. As far as the HNF-1 $\alpha$ gene is concerned, both loss- 
of-function and gain-of-function mutants seem to be associated with diabetes. Recently, over-expression of HNF-1 $\alpha$ was found to negatively regulate the expression of the $H N F-4$-dependent gene lacking HNF-1 $\alpha$ binding sites in their promoter region [17]. We think it is possible that gain-of-function mutants of HNF-1 $\alpha$ also affect HNF-4 dependent gene expression. Functional changes by mutations in the $H N F-4 \alpha$ gene are known to cause MODY1 [18]. The repression of HNF-4 dependent genes by gain-of-function of HNF- $1 \alpha$ could therefore be one of the possible explanations for diabetes in our subjects with T521I and V617I mutations. Another possible explanation might be the inhibition of insulin promoter factor 1 (IPF-1) dependent insulin gene expression as a negative regulator by HNF-1 $\alpha$ [19]. In contrast, HNF-1 $\alpha$ might act as essential positive regulators for the high-level transcription of the human HNF-4 $\alpha$ [20]. Therefore, it is possible that the increase in HNF- $1 \alpha$ activity can disrupt the interrelation between HNF- $1 \alpha$ and HNF- $4 \alpha$, and could eventually inhibit IPF-1 activity. Thus both increased and decreased activity of HNF1 $\alpha$ could have pathophysiologic consequences on normal betacell function by altering the expression of target genes in beta cells $[9,21]$. However exact mechanisms by which the T521I and V617I mutations of the HNF-1 $\alpha$ gene lead to the development of diabetes mellitus should be clarified in the future.

A great phenotypic variability (age at diagnosis, insulin secretion and therapy) was observed both within family members and between different families with the same (L518P519fsTCC $\rightarrow$ A, the A site of the $H N F-1 \alpha$ promoter $+102-$ to- $C$ ) mutation, suggesting that other factors contribute to the phenotype. These factors include environmental influences and genetic modifiers (such as insulin resistance or dyslipidaemia) [22]. When HNF-1 $\alpha$ function is suppressed in the beta cells of transgenic mice, males with mutant protein become overtly diabetic within 6 weeks of age, whereas females just show impaired glucose tolerance [23]. The hormonal profile of the females probably explains this protection from diabetes [24, 25]. It is also possible that additional mutations in other genes could contribute to phenotypic variability.

In conclusion, we identified one known and three novel $H N F-1 \alpha$ mutations in Japanese patients with early-onset diabetes or with a strong family history for diabetes. The overall prevalence rate in the $H N F-1 \alpha$ gene mutation was $10 \%$ in the Okinawa area. The L518P519fsTCC $\rightarrow$ A mutation showed reduced transcription activation in an in vitro functional assay, whereas both the T521I and V617I mutations had increased transcription. Six subjects in three unrelated families were identified with the same L518P519fsTCC $\rightarrow$ A mutation, but their clinical features (age at diagnosis, insulin secretion, and therapy) showed a great variability. Both the T521I and V617I mutations were identified in two related diabetic pa- tients. Precise mechanisms by which these mutations could lead to diabetes mellitus need to be investigated further. Since the prevalence of these mutations is relatively high, the $H N F-1 \alpha$ gene needs to be screened for mutations in patients either with early-onset diabetes or with a strong family history for diabetes irrespective of the presence of anti-GAD 65 antibody.

Acknowledgements. We express our sincere gratitude to Dr. K. Yamagata (Osaka University, Osaka, Japan) for providing the cDNA for human $H N F-1 a \alpha, T T R$-pGL3.

\section{References}

1. Hattersley AT (1998) Maturity-onset diabetes of the young: clinical heterogeneity explained by genetic heterogeneity. Diabet Med 15:15-24

2. Emens LA, Landers DW, Moss LG (1992) Hepatocyte nuclear factor $1 \alpha$ is expressed in a hamster insulinoma line and transactivates the rat insulin I gene. Proc Natl Acad Sci USA 89:7300-7304

3. Takeda J, Kayano T, Fukumoto H et al. (1993) Organization of the human GLUT2 (pancreatic $\beta$ cell and hepatocyte) glucose transporter gene. Diabetes 42:773-777

4. Vaulont S, Puzenat N, Levrat F et al. (1989) Proteins binding to the liver-specific pyruvate kinase gene promoter: a unique combination of known factors. J Mol Biol 20:205-219

5. Noguchi T, Yamada K, Yamagata K et al. (1991) Expression of liver type pyruvate kinase in insulinoma cells: involvement of LF-B1 (HNF1). Biochem Biophys Res Commun 181:259-264

6. Miquerol L, Lopez S, Cartier N et al. (1994) Expression of the L-type pyruvate kinase gene and the hepatocyte nuclear factor 4 transcription factor in exocrine and endocrine pancreas. J Biol Chem 269:8944-8951

7. The Expert Committee on the Diagnosis and Classification of Diabetes Mellitus (1997) Report of the expert committee on the diagnosis and classification of diabetes mellitus. Diabetes Care 20:1183-1197

8. Yamagata K, Oda N, Kaisaki PJ et al. (1996) Mutation in the hepatocyte nuclear factor- $1 \alpha$ gene in maturity-onset diabetes of the young (MODY3). Nature 384:455-458

9. Yoshiuchi I, Yamagata K, Iwahashi H et al. (1999) Three new mutations in the hepatocyte nuclear factor- $1 \alpha$ gene in Japanese subjects with diabetes mellitus: clinical features and functional characterization. Diabetologia 42:621626

10. Kobayashi T, Itoh T, Kosaka K et al. (1987) Time course of islet cell antibodies and $\beta$ cell function in non-insulindependant stage of type 1 diabetes. Diabetes 36:510517

11. Doria A, O'Keeffe C, Yang Y et al. (1999) Phenotypic characteristics of early-onset autosomal-dominant type 2 diabetes unlinked to known maturity-onset diabetes of the young (MODY) genes. Diabetes Care 22:253-261

12. Frayling TM, Bulman MP, Ellard S et al. (1997) Mutations in the hepatocyte nuclear factor 1 alpha gene are a common cause of maturity-onset diabetes of the young in the United Kingdom. Diabetes 46:720-725

13. Kaisaki PJ, Menzel S, Linder T et al. (1997) Mutation in the hepatocyte nuclear factor- $1 \alpha$ gene in MODY and earlyonset NIDDM: evidence for a mutational hot spot in exon 4. Diabetes 45:528-535 
14. Frayling TM, Bulman MP, Appleton M et al. (1997) A rapid screening method for hepatocyte nuclear factor 1 alpha frameshift mutations: prevalence in maturity-onset diabetes of the young and late-onset non-insulin dependant diabetes. Hum Genet 101:351-354

15. Yamada S, Tomura H, Nishigori H et al. (1999) Identification of mutations in the hepatocyte nuclear factor- $1 \alpha$ gene in Japanese subjects with early-onset NIDDM and functional analysis of the mutant proteins. Diabetes 48:645-648

16. Carlomagno F, Melillo RM, Visconti R et al. (1998) Glial cell line-derived neutrophlic factor differentially stimulates ret mutants associated with the multiple endocrine neoplasia type 2 syndromes and Hirschsprung's disease. Endocrinology 139:3613-3619

17. Ktistaki E, Talianidis L (1997) Modulation of hepatic gene expression by hepatocyte nuclear factor 1 . Science 277:109-112

18. Yamagata K, Furuta H, Oda N et al. (1996) Mutation in the hepatocyte nuclear factor- $4 \alpha$ gene in maturity-onset diabetes of the young (MODY1). Nature 384:458-460

19. Yamakawa K, Yamasaki H, Ozaki M et al. (2001) Hepatocyte nuclear factor-1 alpha inhibits insulin promoter factor 1-dependent transactivation of the human insulin gene. Endocr Res 27:63-74
20. Hatzis P, Talianidis I (2001) Regulatory mechanism controlling human hepatocyte nuclear factor $4 \alpha$ gene expression. Mol Cell Biol 21:7320-7330

21. Vaxillaire M, Rouard M, Yamagata K (1997) Identification of nine novel mutations in the hepatocyte nuclear factor 1 alpha gene associated with maturity-onset diabetes of the young (MODY3). Hum Mol Genet 6:583-586

22. Ellard S (2000) Hepatocyte nuclear factor 1 alpha (HNF-1 $\alpha$ ) mutations in maturity-onset diabetes of the young. Hum Mutat 16:377-385

23. Hagenfeldt KA, Herrera PL, Wang $\mathrm{H}$ et al. (2001) $\beta$ celltargeted expression of a dominant-negative hepatocyte nuclear factor- $1 \alpha$ induces a maturity-onset diabetes of the young (MODY)3-like phenotype in transgenic mice. Endocrinology 142:5311-5320

24. Efrat S (1991) Sexual dimorphism of pancreatic $\beta$-cell degeneration in transgenic mice expressing an insulin-ras hybrid gene. Endocrinology 128:897-901

25. Thomas MK, Devon ON, Lee JH et al. (2001) Development of diabetes mellitus in aging transgenic mice following suppression of pancreatic homeoprotein IDX-1. J Clin Invest 108:319-329 